J3eA - Vol. 2 - 4 (2003).

DOI : 10.1051/bib-j3ea:2003004

\title{
Visualisation interactive pour l'étude de la propagation des ondes électromagnétiques
}

\author{
F. Mora * ${ }^{*}$ R. Vauzelle ${ }^{* *}$ et P. Nehlig ${ }^{* * *}$ (Université de Poitiers) \\ Mis en ligne le 17 mars 2003.
}

\begin{abstract}
Résumé
Cet article présente un outil d'aide à la compréhension des mécanismes de propagation des ondes électromagnétiques dans un environnement donné. Plus précisément, il s'agit d'un logiciel interactif qui constitue un support de cours permettant, grâce à de nombreuses animations, de visualiser :
\end{abstract}

- l'évolution spatio-temporelle d'une onde en s'appuyant sur la propagation de son front d'onde ;

- la déformation de ce front lors d'interactions avec des objets (réflexion sur des faces et diffractions sur des arêtes);

- le phénomène d'interférences.

Ce logiciel permet notamment d'illustrer des problèmes liés à l'interface radio pour les systèmes mobiles. Dans ce contexte, au département EEA de la Faculté des Sciences de Poitiers, nous l'utilisons en complément d'un simulateur de canal de propagation par trajets multiples.

Mots-clés : propagation d'ondes électromagnétiques, diffraction, réflexion, interférences, front d'onde, animation, support de cours EEA.

(C) EDP Sciences, 2003.

Niveau de connaissances requis. Connaissances de base en électromagnétisme (niveau DEUG, classes préparatoires).

Niveau des étudiants. Deuxième cycle (maîtrise EEA).

* Frédéric Mora ${ }^{1}$ est doctorant au laboratoire IRCOM-SIC de l'université de Poitiers.

** Rodolphe Vauzelle ${ }^{1}$, maître de conférences, habilité à diriger les recherches, est responsable de la Maîtrise EEA à la Faculté des Sciences de Poitiers.

o e-mail : vauzelle@sic.univ-poitiers.fr (auteur correspondant)

*** Philippe Nehlig ${ }^{1}$ est maître de conférences, responsable du DESS CLABD (Conception Logicielle et Applications aux Bases de Données) de l'université de Poitiers.

\footnotetext{
${ }^{1}$ Département EEA, Université de Poitiers, Bâtiment SP2MI, Boulevard Marie et Pierre Curie, Téléport 2, BP 30179, F-86962 Futuroscope Cedex, France.
} 


\section{Motivations}

Les équations de Maxwell et l'optique géométrique constituent deux approches majeures pour expliquer la propagation des ondes électromagnétiques et les phénomènes qui lui sont liés.

Si les équations de Maxwell décrivent entièrement la propagation d'une onde, elles ne fournissent pas de traitement explicite des phénomènes sous-jacents tels que la réflexion, la diffraction ou encore les interférences. L'étudiant éprouve donc des difficultés pour appréhender ces notions abstraites.

Rappelons que la réflexion et la diffraction sont des phénomènes liés aux interactions qu'une onde peut avoir avec l'environnement dans lequel elle évolue. Le premier se manifeste lorsqu'une onde rencontre une surface (voir partie 2.1.2), le second lorsqu'elle rencontre une arête ou un sommet (voir partie 2.1.3). Dans les deux cas, on peut considérer que ces phénomènes entraînent l'apparition d'ondes secondaires dont les caractéristiques de formes et de direction dépendent de la nature et des propriétés des obstacles rencontrés.

Quant au phénomène d'interférences, il résulte de la combinaison de différentes ondes. Il engendre des noeuds et des ventres d'amplitude de l'onde résultante en fonction du point d'observation, dus à des interférences constructives ou destructives entre ondes. Nous reviendrons plus en détail sur ce phénomène dans la partie 2.1.4.

Il est courant de considérer l'optique géométrique et le concept associé de rayon pour décrire ces phénomènes. Mais, en assimilant une onde à un rayon, on introduit un certain nombre d'éléments déroutants pour l'étudiant. En effet, la notion de rayon à laquelle on associe naturellement une direction de propagation contraste avec celle de surface d'onde ou de front d'onde.

Notre but est de faire cohabiter sur un même support des notions liées à ces deux théories. Nous en tirons une série d'images et de vidéos offrant une description explicite des mécanismes de propagation d'ondes électromagnétiques sans introduire les contradictions apparentes évoquées précédemment [1]. Utilisées en cours, elles offrent un riche support pédagogique et permettent d'illustrer l'évolution spatio-temporelle d'une onde.

\section{Propagation des ondes électromagnétiques}

Dans cette partie, nous présentons les principes sur lesquels repose notre logiciel, conjointement aux images et animations que nous pouvons en extraire. Nous nous plaçons dans le cas d'une propagation non guidée, dans des environnements 2D dont les éléments sont représentés par des polygones aux côtés parfaitement réfléchissants. En positionnant une source ponctuelle dans une telle scène, nous proposons d'y représenter l'évolution des fronts d'onde et l'amplitude associée dans le temps et l'espace.

Certains outils informatiques sur la propagation des ondes électromagnétiques reposent sur une discrétisation des équations de Maxwell. Dans notre cas, nous utilisons une discrétisation de leur solution. Par ailleurs, nous représentons une onde à travers son front d'onde qui est alors modélisé par un cercle, ou un arc de cercle. La propagation de l'onde est visualisée par tracés successifs de cercles concentriques. Pour effectuer la discrétisation des fronts d'ondes, nous nous basons sur des propriétés issues de la géométrie discrète et notamment les cercles analytiques discrets [2], dont les tracés concentriques forment un pavage du plan.

\section{1. Évolution des fronts d'onde directe, réfléchie et diffractée}

\subsubsection{Onde directe}

Sur ces bases, une source ponctuelle $S$ rayonne une onde que l'on peut visualiser grâce au front d'onde qu'elle génère. Il est représenté par des cercles concentriques de rayon croissant pour traduire son évolution spatiotemporelle. Les figures 1 et 2 montrent la propagation de ce front tant qu'il ne rencontre pas d'obstacle. 


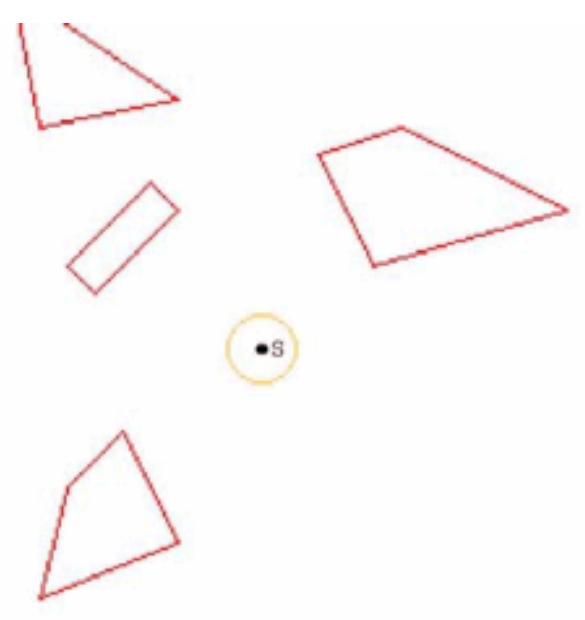

Fig. 1. Propagation d'une onde directe, instant $t_{1}$.

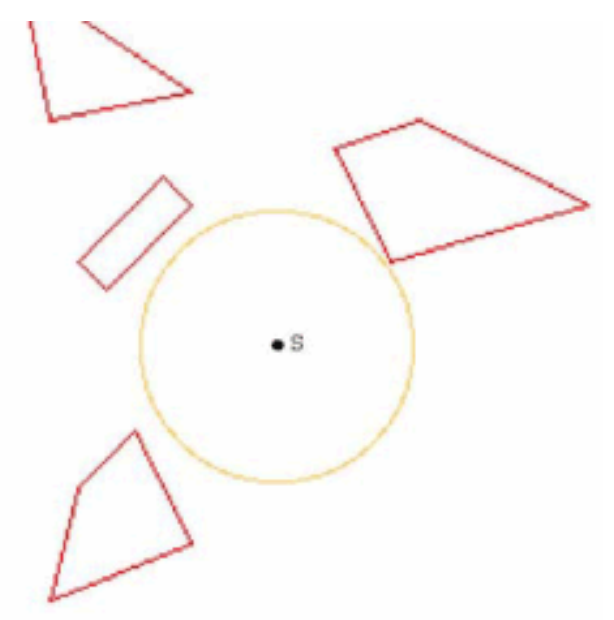

Fig. 2. Propagation d'une onde directe, instant $t_{2}>t_{1}$.

\subsubsection{Onde réfléchie}

Cette représentation explicite des fronts d'onde implique un traitement des interactions qu'ils subissent au cours de leur propagation. Nous nous penchons dans un premier temps sur le phénomène de réflexion, sans considérer la diffraction. Pour modéliser une interaction de type réflexion, nous nous appuyons sur l'optique géométrique.

La première loi de Descartes permet de déterminer les caractéristiques de l'onde réfléchie. Pour définir la direction de propagation de l'onde réfléchie, on considère le principe de la source image qui détermine une source virtuelle comme étant le symétrique de la source réelle par rapport à la surface réfléchissante (Fig. 3).

Le front d'une onde réfléchie se modélise alors par un cercle ou arc de cercle dont le centre est une source virtuelle telle que nous venons de l'expliquer.

En poursuivant l'évolution du front d'onde de la figure 2, celui-ci rencontre les côtés de polygones représentant l'environnement. Il donne alors naissance à des fronts d'ondes réfléchies définis par des arcs de cercles (Fig. 4). 


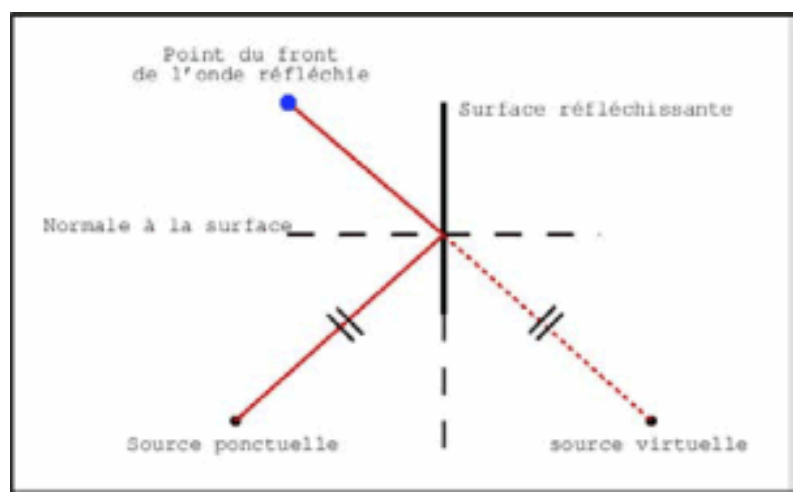

Fig. 3. Principe du phénomène de réflexion.

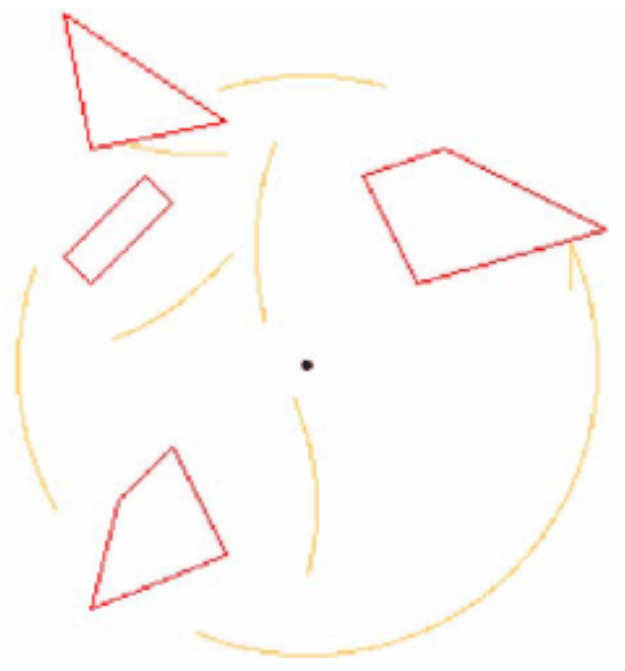

Fig. 4. Illustration du phénomène de réflexion.

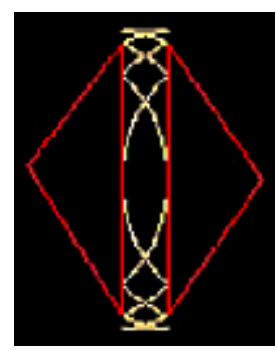

Animation 1. Illustration du phénomène de réflexion.

On peut souligner que la réflexion permet de couvrir une zone qui n'est pas en visibilité directe de la source, comme c'est le cas pour l'onde réfléchie sur le polygone en haut à gauche de la scène. L'animation 1 donne encore une meilleure illustration du phénomène de réflexion. 


\subsubsection{Onde diffractée}

Pour compléter la présentation des phénomènes de propagation d'une onde, il est nécessaire d'introduire la diffraction. C'est sur elle que nous focalisons maintenant notre attention, ignorant cette fois-ci le phénomène de réflexion. La diffraction peut s'expliquer par le principe de Huygens-Fresnel. Il indique qu'une pointe illuminée par une onde se comporte comme une nouvelle source, réémettant une onde secondaire.

Comme l'illustre la figure 5, l'onde diffractée peut ainsi être représentée par son front d'onde dont le centre virtuel est le sommet de la pointe illuminée.

Notre logiciel intègre ce principe et permet de produire une image comme celle de la figure 6 sur laquelle la diffraction est prise en compte. On y montre que le sommet du polygone se comporte comme une source secondaire. En outre, l'étudiant voit bien que la zone entre les deux triangles n'aurait pas pu être atteinte sans ce phénomène, qui n'est pas prévu par l'optique géométrique.

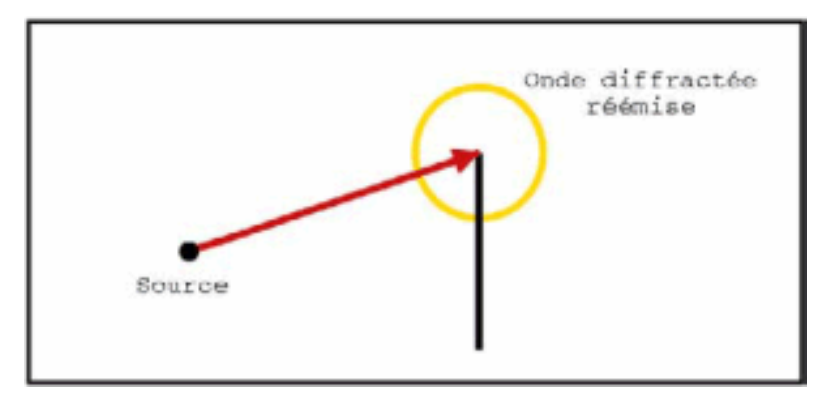

Fig. 5. Principe du phénomène de diffraction.

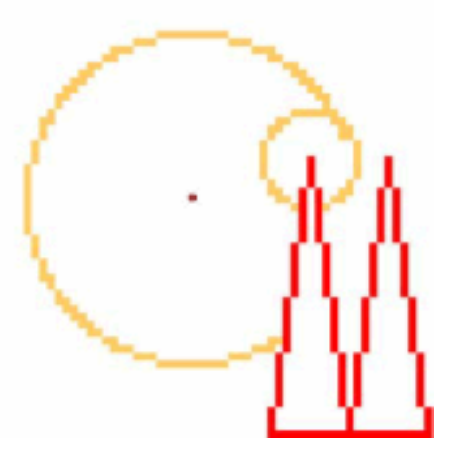

Fig. 6. Illustration du phénomène de diffraction.

La figure 7 présente l'évolution du front un peu plus tard dans le temps, de façon à montrer l'existence de la deuxième diffraction sur le deuxième triangle qui permet de couvrir des zones encore moins accessibles à l'onde directe. On peut également apprécier sur ces deux images la combinaison des différents fronts d'ondes, tous tangents entre eux. Enfin, la figure 8 montre le phénomène de diffraction sur la scène ou nous avons déjà pu apprécier la propagation d'une onde directe et réfléchie séparément. 


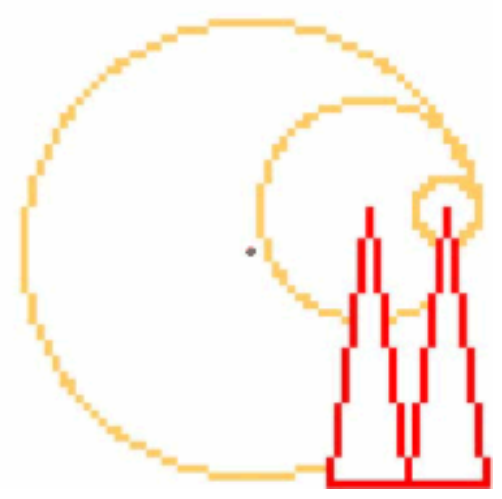

Fig. 7. Illustration d'une diffraction subie par l'onde diffractée sur la première pointe.

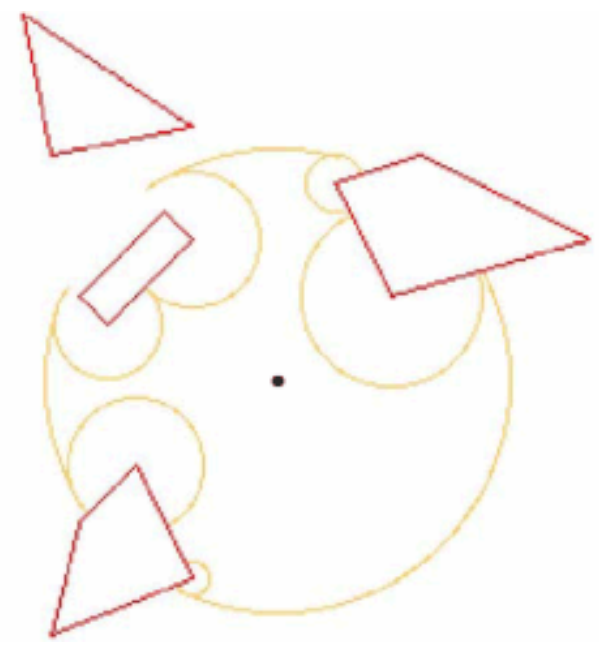

Fig. 8. Diffraction multiple d'une onde. 


\subsubsection{Association des phénomènes de réflexion et de diffraction}

Nous présentons ici deux images d'une propagation prenant en compte la réflexion et la diffraction. Prises à des instants $t_{1}$ (Fig. 9) et $t_{2}$ (Fig. 10), $t_{1}<t_{2}$, on y observe les fronts d'ondes directe, réfléchies et diffractées.

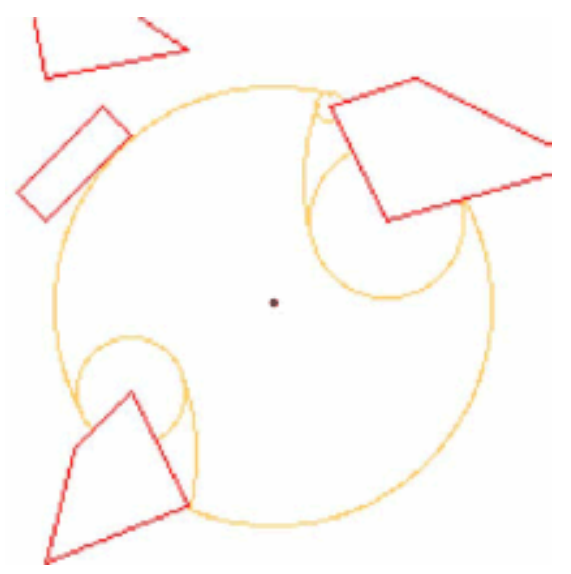

Fig. 9. Propagation des ondes directe, réfléchies et diffractées à l'instant $t_{1}$.

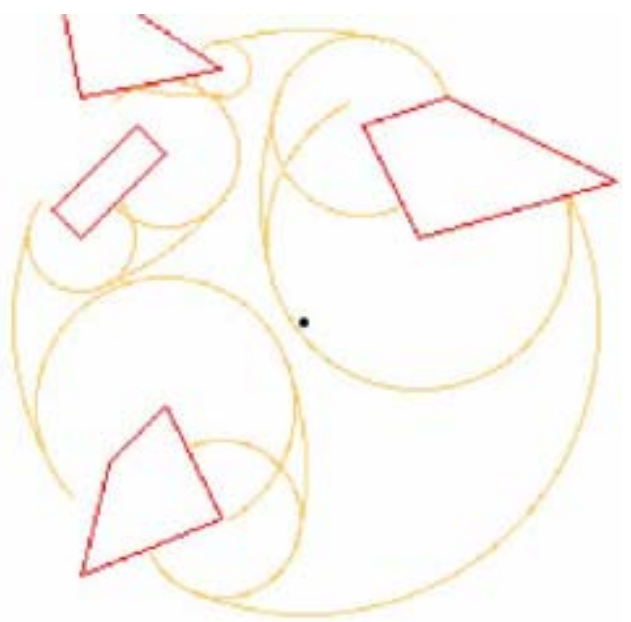

Fig. 10. Propagation des ondes directe, réfléchies et diffractées à l'instant $t_{2}$.

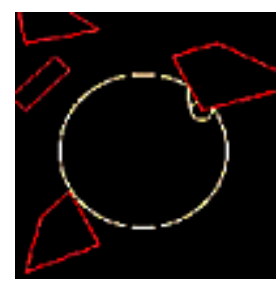

Animation 2. Réflexions et diffractions liées à la propagation d'une onde électromagnétique.

L'animation 2 dont sont extraites ces deux images permet de donner à l'étudiant une vision complète des mécanismes de propagation des ondes électromagnétiques. Dans le cas présent, seule l'onde directe est sujette à des réflexions et des diffractions. Pourtant, on peut déjà noter la prolifération des fronts d'ondes secondaires, qui, on peut l'introduire à ce niveau, va engendrer de multiples interférences. 


\subsection{Aspect énergétique}

Ce paragraphe présente l'aspect énergétique de la propagation des ondes. Nous mettons en évidence le phénomène d'interférences en modélisant l'amplitude associée aux fronts d'onde décrits dans le paragraphe précédent. Dans ce but, nous nous appuyons sur les solutions des équations de Maxwell en espace libre :

$$
u(r, t)=\frac{E_{0}}{\tau} \cos (w t-k r)
$$

$$
u(r, t) \quad E_{0}
$$

avec représentant l'amplitude du champ électrique ou magnétique associé à une onde. est l'amplitude initiale de ce champ, $r$ la distance de la source au point d'observation, $w$ la pulsation de l'onde et $k$ le vecteur d'onde qui lui est associé.

Ainsi, à un instant $t$ donné, on évalue les amplitudes en tout point de la scène ayant été balayé par un ou plusieurs fronts d'onde (Fig. 11).

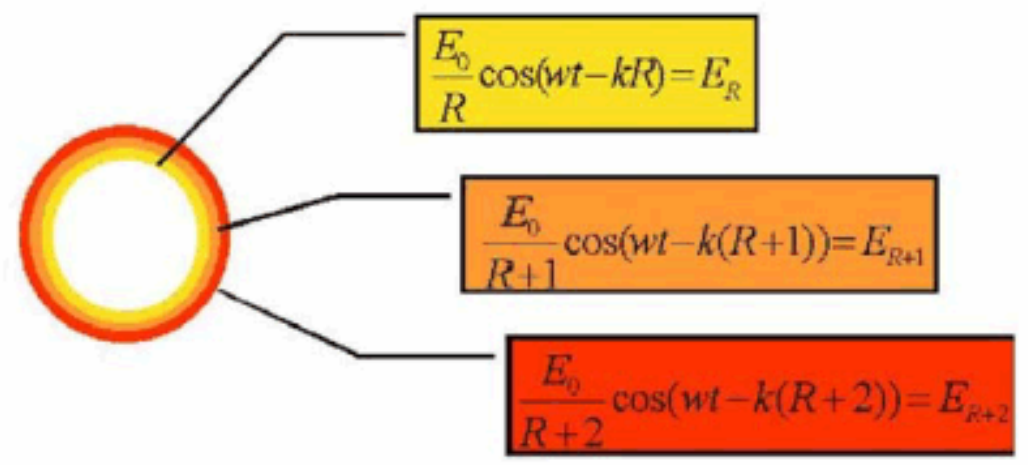

Fig. 11. Calcul de l'amplitude.

La représentation des valeurs obtenues se traduit par une échelle de couleur comme le montre la figure 12. Les zones bleues symbolisent les plus basses amplitudes et les zones rouges les plus fortes.

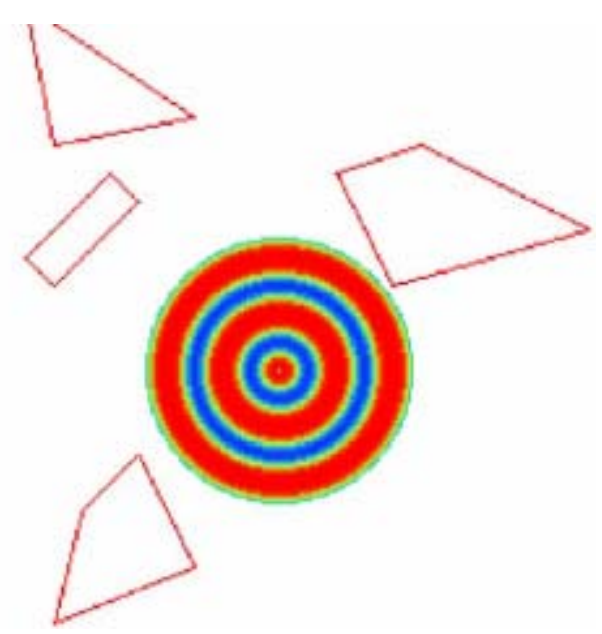

Fig. 12. Évolution de l'amplitude. 


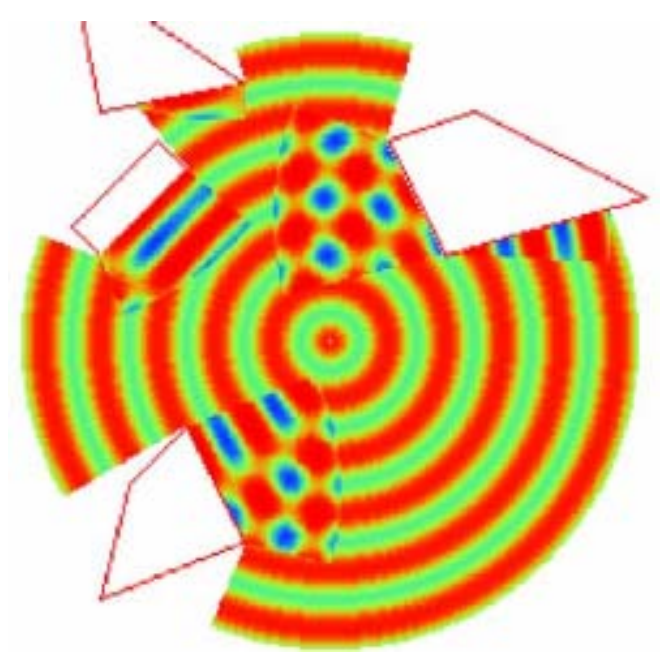

Fig. 13. Interférences onde directe - ondes réfléchies.

La visualisation du phénomène d'interférence est montrée sur la figure 13. Nous nous situons un peu plus tard dans le temps par rapport à la figure 12, et nous voyons apparaître des zones atteintes par l'onde directe et des ondes réfléchies. Au sein de ces zones apparaissent les interférences constructives et destructives, liées à la superposition des ondes.

L'établissement des interférences dans le temps est mis en avant dans les animations 3 et 4 . La première reprend le même environnement que l'animation 1 et permet de bien établir le parallèle entre la visualisation des fronts d'onde et l'aspect énergétique. Notons que la figure 14 est extraite de la deuxième animation. On peut souligner que les zones de basses et de hautes amplitudes obtenues n'étaient pas prévisibles initialement.

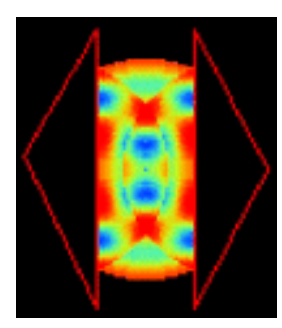

Animation 3. Illustration du phénomène d'interférences.

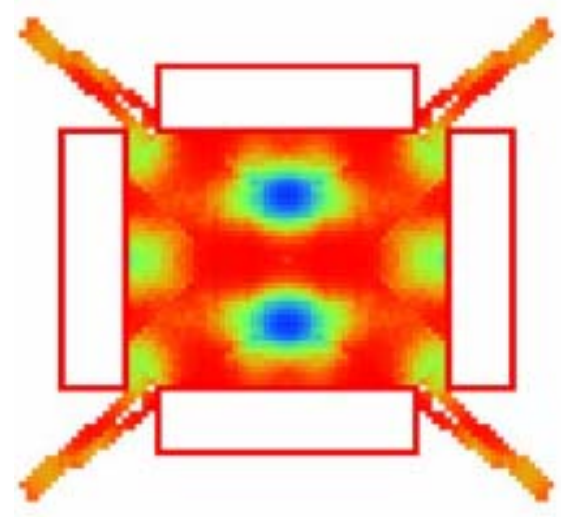

Fig. 14. Interférences multiples. 


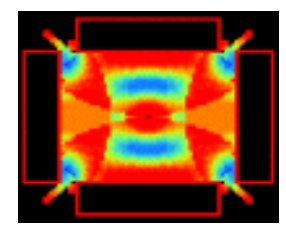

Animation 4. Évolution des interférences dans le temps.

De plus, l'animation 4 montre l'apparition de deux zones de très basses amplitudes qui sont pourtant en visibilité directe de la source placée au centre de la scène. On met ainsi en avant la difficulté de prévoir le comportement des ondes électromagnétiques. On peut faire le lien avec les enjeux industriels actuels en matière de radiocommunications, qui consistent à prévoir l'emplacement optimal des stations de base des réseaux mobiles.

\section{Conclusion}

Cet ensemble d'images et de vidéos offre un support pédagogique attrayant pour l'étudiant. Leur simple observation contribue à lui faire comprendre la propagation des ondes électromagnétiques et les principaux phénomènes sous-jacents.

De plus, le logiciel présenté peut aussi permettre d'illustrer les difficultés de mise en oeuvre des réseaux mobiles et ainsi servir d'introduction à des cours pourtant sur l'étude de l'interface radio de ces systèmes. Dans ce cadre, au département EEA de la Faculté des Sciences de Poitiers, nous utilisons ce logiciel comme complément d'un simulateur de canal de propagation par multi-trajets.

Notons enfin, que ce logiciel fait l'objet d'un développement approfondi au laboratoire IRCOM-SIC de l'Université de Poitiers. Une version plus aboutie verra prochainement le jour ; elle revêtira la forme d'un logiciel utilisable en TP, permettant à l'étudiant de placer lui-même obstacles et sources d'émission dans la scène et d'apprécier en temps réel la propagation des ondes.

\section{Références bibliographiques}

[1] F Mora, Géométrie discrète et propagation d'ondes : étude de faisabilité, Rapport de DEA, Université de Poitiers (2002).

[2] E. Andres et M.-A. Jacob, The discrete Analytical Hyperspheres, IEEE Transaction on visualization and computer graphics, 3 (January-march 1997) 1. 